\title{
VIRTUAL FARMER: CONTROLLING PHYTOCHROME SIGNALING IN PLANTS THROUGH CYBER-PHYSICAL SYSTEM
}

\author{
ANKUSH RAI*, JAGADEESH KANNAN R \\ School of Computing Science and Engineering, VIT University, Chennai, Tamil Nadu, India. Email: ankushressci@gmail.com
}

Received: 13 December 2016, Revised and Accepted: 03 April 2017

\section{ABSTRACT}

Objective: Under external environment stimuli, seedlings undergo variation of morphology and alterations in their genetic sequences. Phytochrome signaling, i.e., feedback reaction of plants to photons and other nutrient cycle plays a crucial role in its maturation.

Method: In this research work, we create a cyber-physical system to control such morphogenesis of plants through the help of artificial intelligence framework which identifies and control the crucial feedback between plants' genetic transcription with respect to the external stimuli such as nutrients, electricity, and magnetism.

Results and Discussion: This leads a plant to autonomously grow without its disadvantageous traits by destabilizing its negatively acting transcriptional regulators and enhance the plant's advantageous features by controlling its positively acting transcriptional regulators. This has led us to control the plant metabolism, plant growth without soil, manipulate the immunity of plant against disease, develop a plant metabolic profile, and maximize its yield deprived off from its seasonal attribute.

Keywords: Epigenetic transcription, Cyber-physical system, Artificial intelligence.

(C) 2017 The Authors. Published by Innovare Academic Sciences Pvt Ltd. This is an open access article under the CC BY license (http://creativecommons. org/licenses/by/4. 0/) DOI: http://dx.doi.org/10.22159/ajpcr.2017.v10s1.19746

\section{INTRODUCTION}

There is an increasing need to recirculate and reuse nutrient solutions with a specific end goal to diminish environmental and monetary expenses in hydroponic system [1-4]. However, the advances in hydroponic system caused the following problems:

1. One of the weakest points in hydroponics is the absence of qualitative information on managing nutrient solution. Numerous cultivators and research scientists dump out nutrient solutions and refill at week after week interims. Dumping and supplanting solution on iterative basis is superfluous. Monitoring ions in solution is not always necessary. The quick consumption of a few nutrients regularly makes individuals add poisonous measures of nutrients to the solution. Monitoring ions in hydroponic solution is an intriguing aspect, yet it is not a compelling process for effective nutrient management [5-8].

2. Second, due to the absence of soil in hydroponic system, there emerges the issue of root and its hair growth to empower viable absorption of nutrients. The hydroponic buffer solution neglected to instigate root hairs on the roots of onion, corn, and so on. Further, this causes lower respiration rates of the plants' root and it ascends as biomass with algae as the age of plants increments. In this manner, the respiration rate of plants gets affected with higher $\mathrm{CO}_{2}$ fixation in hydroponic system. The oxygen concentration is subject to the gasfluid interface framed on the upper surface of the hydroponic fluid, as given by Henry's law, contingent upon weight and temperature conditions. Respiration rates of the plants diminish in an exponential way with plant age and microbial movement, consequently bringing on inefficient yields in hydroponic system [9-13].

In this research project, we propose a cyber-physical system (CPS)based environment to analyze the nutrient uptake and metabolic process in plants to control their transcriptional regulators in addition with enhanced plant root growth through electrical and hydroscopic incitement to give a computationally invigorated root growth environment [14-18]. This controls the actuation of root growth and root hair development in accordance with computer- controlled nutrient management module. This system will improve the root respiration and proficient management of $\mathrm{O}_{2}$ and $\mathrm{CO}_{2}$ in closed hydroponic environment to empower high root respiration rates, effective absorption of nutrients, and give higher yield.

\section{METHODS}

During the past two decades, nutrients have been managed manually in closed hydroponic systems according to the principle of "mass balance," which implies that the mass of nutrients is either in solution or in plants. The nutrients in the hydroponic solution can be divided into three groups with characteristics based on how quickly they are removed from solution. Plants quickly remove their everyday apportion of a few nutrients while different nutrients settle down to accumulate in the solution. This implies that the concentrations of nitrogen, phosphorous, and potassium can be at low levels in the solution $0.1 \mathrm{mM}$ or a few ppm) after absorption because these nutrients are already absorbed in the plant. However, in the next refill state, ungauged nutrient in the hydroponic solution will impart from nutrients to the plants than it is required, leading to harmful effects in plant growth. Here, phosphorous has to be maintained at minimum $0.5 \mathrm{mM}$ in the recirculating solution, the concentration of phosphorous in the plant could increase to $1 \%$ of the dry mass, which is 3 times higher in comparison to the optimum concentration in most plants. This high phosphorous level can induce iron and zinc deficiency. Maintaining high concentrations of nutrients in the solution can bring about exorbitant uptake that can lead to nutrient imbalances. Low concentrations in the refill solution are difficult to monitor and control that should be mandatorily maintained in the circulating solution. The errors in measurements, for $\mathrm{K}, \mathrm{P}$, and $\mathrm{Mn}$ can be 10 times higher because the solution levels are lower. Owing to the differential rate of nutrient uptake, conductivity measurements mostly measure the calcium, magnesium, and sulfate remaining in solution. The contribution of micronutrients remains $<0.1 \%$ to electrical conductivity. Therefore, spending efforts in sensing nutrient content in hydroponic solution is fruitless, as it does not impart any contribution in deciding the next consecutive recipe of refill concentration of nutrients in the hydroponic solution $[19,20]$. It is thus important to comprehend 


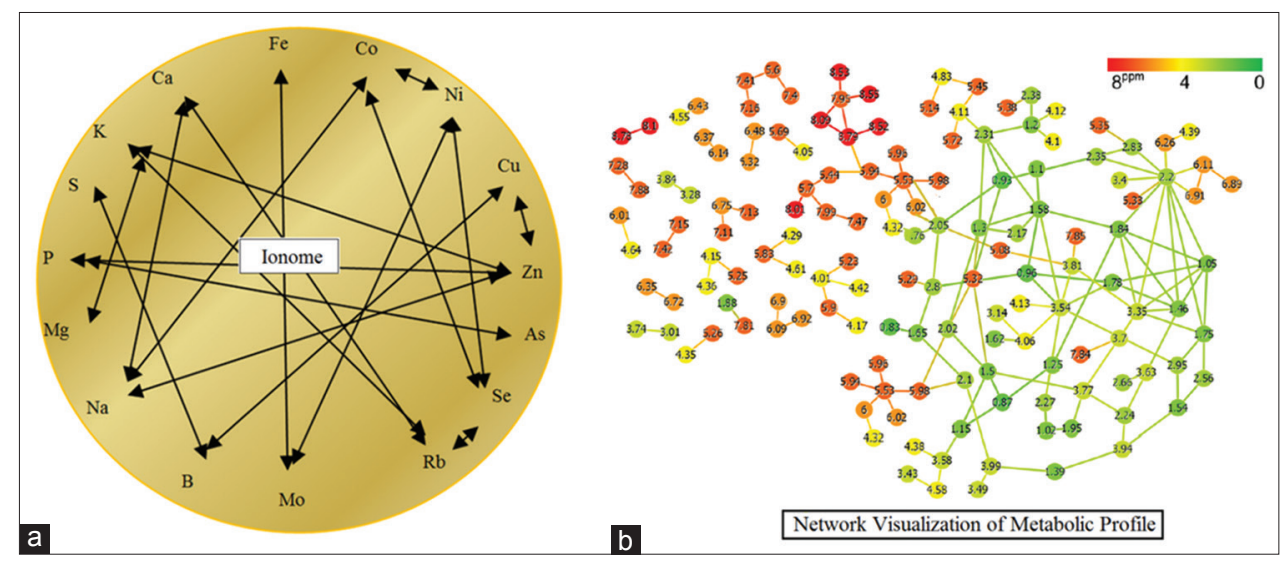

Fig. 1: (a) Near-infrared transmission spectroscopy of plants revealing chemical interactions between nutrient elements with essential, beneficial, and toxic elements. (b) With the help of dynamic Boolean networks and the processing of information corresponding to elemental interactions with the concentrations, we developed a metabolic profile of the plant tissue

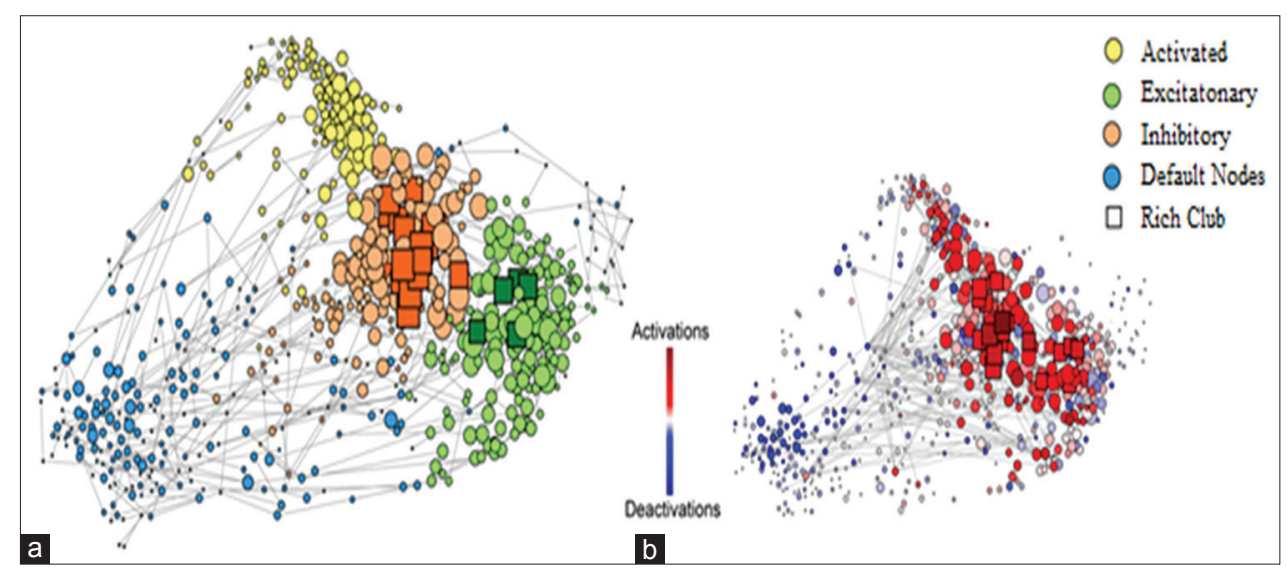

Fig. 2: Topological representation of the functional coactivation network. (a) Force-based layout of the minimum spanning tree is used to locate nodes in relation to their topological (rather than anatomical) proximity to each other. Different modules are coded by color, and rich-club nodes are represented by squares, with the size of all nodes proportional to their weighted degree (strength). (b) Nodes in anatomical space colored according to proportion of times present activations and deactivations. Nodes arranged in the same layout as a, and colored as in b. Also, note that the rich club concentrates most of the activations, whereas the periphery and particularly the defaultmode network concentrate the deactivations. Edges represent the top 1 percentile of the most consistently reported activations and deactivations (no directions shown for clarity purposes). Edges can be seen spanning across different modules

the standards for nutrient substitution, particularly when the solution is continuously recycled over the life cycle of a crop. It seems that automatic addition of refill solution and effective nutrient circulation system are required to avoid depletion.

Here, the idea is to analyze and alter the nutrient concentration and composition with the growth stage of the plant to decrease nutrient aggregation in the solution. Consequently, to make this method efficient, we also need to solve the problem of root and root hair growth. As root growth primarily occurs during early vegetative growth and is less critical amid late vegetative growth, root growth decreases and even stops during reproductive growth. Analysis and modeling of hydroponic solution is unnecessary, inaccurate, and difficult to interpret, but analysis of plant tissue in association with nutrient absorption through near-infrared spectroscopy for nutrient detection method is useful, accurate, and relatively easy to interpret. The ionome of a plant or plant part comprises all the elements, including essential, beneficial, and in some cases, even toxic elements [21]. The homeostasis of the plant ionome is controlled by a vast network of interactions between the different elements, a subset of which is presented graphically in Fig. 1a. In our preliminary research, the computational modeling experiments focus exclusively on identifying one or a selected few elements, sensitive to alterations in the interactions. Previously, as in most cases, it is not

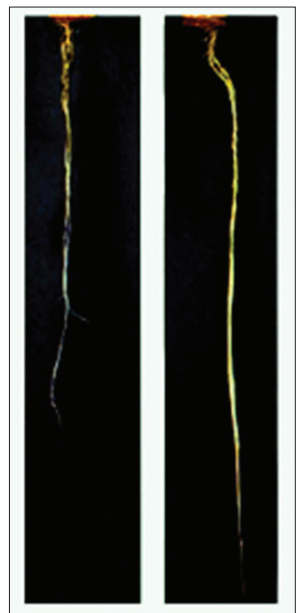

Fig. 3: Output of electrically stimulated enhanced root growth

possible to predict elemental interactions in this giant network, as too many factors of the ionome regulation are still unknown. However, we deployed our own mathematically modeled coactivated dynamic 


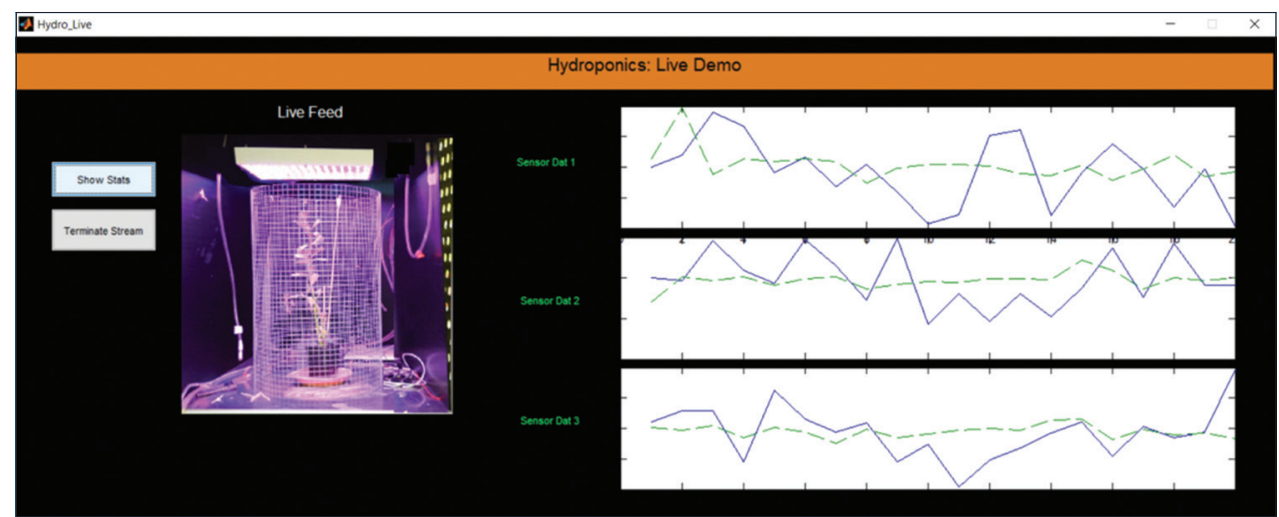

Fig. 4: Demo of the artificial intelligence-controlled framework showing beating all odds of growing orchid in impossible climate settings of Chennai

Boolean networks (DBNs), which give the results in accordance with the experimentally determined elemental interactions (Fig. 1b). The presented approach is more feasible with the developments in multielemental analytical methods as well as easily accessible programs for multivariated investigation to outline the profile of metabolic system. This overview of the plant ionome will enable nutrient management in consequent refill of hydroponic solution, diagnosing physiological and biochemical changes within the plant, by the utilization of ionomics, biomarkers for nutrient imbalances as well as other biotic and abiotic stresses can be found. In addition, it might serve as a less expensive and quicker technique for phenotyping mutants compared. Further research in this area will attract growing attention in automated hydroponic systems [22,23].

In DBN, coactivation network was topologically complex in several ways. The nodal degree distribution was fat-tailed with high-degree hub nodes to be located in presented polymorphic DBN using sequence of information to excite the necessary regions and access the information in an associative form [24-26]. This enables the machine not only to learn but also enables it to embark the cross relationship between various data for prediction or simulation-based logical conclusion. Physically, this topology was embedded parsimoniously, in terms of the connection distance between coactivated nodes (Fig. 2). Most connections or edges were separated by short sequence of excitatory data, significantly shorter than random networks; with $\mathrm{p}<10^{-3}$ in the permutation test. Relatively few edges were of long distance and these were often interhemispheric projections between bilaterally homotopic regions where $14 \%$ of longest connections (defined as top 10 percentile) were homotopic, significantly more than random.

Although the network cost was overall low, as measured by the distance of connections, the network topology still managed to balance integration and segregation between all topological artificial neural regions: The clustering of the network threshold at sparse levels was much higher than random, while retaining a similar path length, i.e., it was small world. In all these aspects, the organization of the coactivation network was convergent with properties of a comparable functional connectivity network generated from resting state of excitatory sequences. As known from a prior study, and reproduced here, the polymorphic DBN based neural networks for feature extraction and encoding of it for the feature sets are used), with fat-tailed degree distributions and parsimonious distance distributions between its weighted connectio.

For the coactivation network, it was possible to assign functional as well as anatomical labels to the modules using Boolean networks. To do this, we will consider the high-level behavioral domains used which describe each contrast in the primary literature: Action, cognition, interaction, perception, and interoception. We then labeled each edge according to the domain most frequently causing coactivation of the corresponding pair of regions. In the occipital module, the highest proportion of intramodular modeling is set aside; as once modeled, the polymorph neural network has proven its evolutionary nature and self-modeling in dynamic scenario in previous studies corresponded to coactivation by perception (39\%) and the other domains coactivated $<20 \%$ each; similarly, in the default-mode module, other domains each accounted for $<21 \%$; whereas, in the central module, $62 \%$ of intramodular edges were coactivated by action [27].

The second problem is to computationally stimulate the root growth of the hydroponic plants using electric fields. Root morphology was strongly affected by the electric field applied, and a significant variation in root growth rate was observed along the gradient. Here, the relation between stimulation and change in the parameters of root growth is also modeled with DBN to determine the current stimulation pattern. Fig. 3 shows a pronounced length, root hairs' development, and root branching, compared to the plants grown with negatively charged collar. The preliminary works are shown in Fig. 3.

Thus, it seems reasonable to say that the presented research has firm prominence with its scope fulfillment and is relatively specialized for action. Modeling tasks accounted for approximately the same proportion of intramodular edges account for $34 \%$ and $38 \%$, respectively, and therefore we described it as specialized for other executive functions. This project shall boost the application in modeling metabolic profile and nutrient interactions and provide an intelligent framework for CPS to computationally control nutrient management and root growth pattern (Fig. 4).

\section{CONCLUSION}

The presented application-based research as an outcome from the collaboration is aimed to integrate the presented computational and CPS framework with the generic existing infrastructure of both countries to furnishing the commercial demand of scalable CPS-based hydroponic system in low-cost settings. This is a huge achievement as it will allow farmers to drastically cut cost in crop production, more yield in less area, brings automation in crop growth, and enable farmers to directly connect with the consumers as the web framework allows them to directly sell and lease the food resources to the consumers. It allows the drastic reduction in greenhouse gasses, and farmers can come together to scale the system.

\section{REFERENCES}

1. Chang YS, Song HG, Kim DE. Development of a chain conveyor type row spacing system for plant factory. J Bio Environ Control 2005;14(1):7-14.

2. Chang YS, Kim DE, Kim HH, Kim JG, Yu IH. Utilization effect of sliding type crop row spacing system. J Bio Environ Control 2006;15(1):63-9.

3. Drury G. Apparatus and method for transporting growing plants. U.S. Patent No. 4476651 A; 1984.

4. Edan Y, Han S, Kondo N. Automation in agriculture. In: Nof SY, editor. Springer Handbook of Automation. Berlin, Heidelberg, Germany: 
Springer-Verlag; 2009. p. 1095-128.

5. Haub JT, Krassas JG, Rustad SC, Davis N. Method and apparatus for increasing the spacing between plants in accordance with their growth rate. U.S. Patent No. 4216618 A; 1982

6. Horibe K, Mori K, Matsuo K, Kondoh K, Kameoka T. Studies on the development of plant transfer equipment (part 2). J Jpn Soc Agric Mach 1993;55(1):49-57.

7. Hydroponic Production Lines. Available from: http://www. greenautomation.fi/content/en/11501/857/857.html. [Last accessed on 2014 Dec 23].

8. Ikeda AK, Nakayama S, Ishii T, Itakura I. Apparatus for plant cultivation. U.S. Patent No. 4617755 A; 1986.

9. Jansen WH. System with plant carriers that can be moved over rails. European Patent (EP) No. 1374666 A1; 2004.

10. Kim DO, Ho JO, Yoon JB, Choi KL. Automation in the plant factories with natural lighting. In: The Plant Factory. Republic of Korea: Rural Development Administration (RDA); 2013a. p. 173-89.

11. Kim DO, Ho JO, Yoon JB, Choi KL. Automation in the plant factories with artificial lighting. In: The Plant Factory. Republic of Korea: Rural Development Administration (RDA); 2013b. p. 79-95.

12. Kim YH. Studies on the Automatic System for Plant Factory. In: Proceedings of $4^{\text {th }}$ Urban-Type Plant Factory Symposium. Seoul, Republic of Korea; 2011. p. 125-40.

13. Korea Rural Development Administration (RDA). Development of Plant Production Factory System for Fresh Leaf Vegetables Production all the Year Round. Technical Report; 2005.

14. McHugh R, Zhang HH. Virtual prototyping and mechatronics for $21^{\text {st }}$ century engineering. Int J Eng Res Innov 2011;3(2):69-75.

15. Mobile Gully System (MGS). Available from: http://www.hortiplan. com/site/index.php?menu=3. [Last accessed on 2014 Dec 23].
16. Mori K, Horibe K, Horino Y, Isomura A, Yonekawa Y, Andoh Y, et al. Studies on the development of plant transfer equipment (part 1). J Jpn Soc Agric Mach 1991;55(1):49-57.

17. Rasin B. Device for moving of growing troughs in a growing plant. European Patent (EP) No. 0663791 B1; 1998

18. Roberts DS. Method and apparatus for hydroponic gardening. U.S. Patent No. 4932158 A; 1990.

19. Roodbergen KJ, Vis IF. A survey of literature on automated storage and retrieval systems. Eur J Oper Res 2009;194(2):343-62.

20. Space-O-Mat System. Available from: http:/www.visser.eu/products/ all-products/Space-O-Mat\%20System. [Last accessed on 2014 Dec 23].

21. Ankush R. Application of Artificial Intelligence for Virtually Assisted Prognosis of Diabetes: A NODDS Project. IJCA Proceedings on National Seminar on Application of Artificial Intelligence in Life Sciences. NSAAILS(1):1-5, February; 2013.

22. Rai A, Ramanathan S. Distributed learning in networked controlled cyber physical system. Int J Pharm Technol 2016;8(3):18537-46.

23. Rai A. High performance computing: A reality at Central-India. Int J Innov Res Dev 2013;2(3):734-43.

24. Rai A. Automation in computation over linux integrated environment. J Adv Shell Program 2014;1(1):18-20.

25. Rai A. Air Computing: A parallel computing module for offloading computational workload on neighboring android devices. Recent Trends Parallel Comput 2015;1(3):10-3.

26. Rai A. Parallelizing mutations for genetic algorithm. Recent Trends Parallel Comput 2015;1(3):7-9.

27. Rai A, Ramanathan S, Kannan RJ. Quasi Opportunistic Supercomputing for Geospatial Socially Networked Mobile Devices. Enabling Technologies: Infrastructure for Collaborative Enterprises (WETICE), 2016 IEEE $25^{\text {th }}$ International Conference on IEEE; 2016. 